\title{
Single $\mathrm{P}$ and As dopants in the $\mathrm{Si}(001)$ surface
}

\author{
M. W. Radny ${ }^{\text {a) }}$ and P. V. Smith \\ School of Mathematical and Physical Sciences, University of Newcastle, Callaghan 2308, Australia \\ T. C. G. Reusch \\ Center for Quantum Computer Technology, University of New South Wales, Sydney 2052, Australia \\ O. Warschkow and N. A. Marks \\ Center for Quantum Computer Technology, School of Physics, University of Sydney, \\ Sydney 2006, Australia \\ H. Q. Shi \\ School of Physics, University of Sydney, Sydney 2006, Australia
}

D. R. McKenzie

Centre for Quantum Computer Technology, School of Physics, University of Sydney, Sydney 2006, Australia

S. R. Schofield

School of Mathematical and Physical Sciences, University of Newcastle, Callaghan 2308, Australia

N. J. Curson and M. Y. Simmons

Centre for Quantum Computer Technology, University of New South Wales, Sydney 2052, Australia

(Received 26 June 2007; accepted 28 August 2007; published online 13 November 2007)

\begin{abstract}
Using first-principles density functional theory, we discuss doping of the $\mathrm{Si}(001)$ surface by a single substitutional phosphorus or arsenic atom. We show that there are two competing atomic structures for isolated $\mathrm{Si}-\mathrm{P}$ and $\mathrm{Si}-\mathrm{As}$ heterodimers, and that the donor electron is delocalized over the surface. We also show that the $\mathrm{Si}$ atom dangling bond of one of these heterodimer structures can be progressively charged by additional electrons. It is predicted that surface charge accumulation as a result of tip-induced band bending leads to structural and electronic changes of the $\mathrm{Si}-\mathrm{P}$ and $\mathrm{Si}-\mathrm{As}$ heterodimers which could be observed experimentally. Scanning tunneling microscopy (STM) measurements of the $\mathrm{Si}-\mathrm{P}$ heterodimer on a $n$-type $\mathrm{Si}(001)$ surface reveal structural characteristics and a bias-voltage dependent appearance, consistent with these predictions. STM measurements for the As:Si(001) system are predicted to exhibit similar behavior to P:Si(001). (C) 2007 American Institute of Physics. [DOI: 10.1063/1.2786991]
\end{abstract}

\section{INTRODUCTION}

Phosphorus (P) and arsenic (As) are traditional dopants used in the preparation of $n$-type bulk silicon. Replacing one silicon atom in bulk silicon by a donor atom such as $\mathrm{P}$ or As leads to an additional (donor) electron that is weakly bound to the dopant atom. As a result, the silicon bulk environment becomes negatively charged while the impurity atom is positively charged. Recent work has shown, however, that as the size of the P:Si system reduces, this charge transfer reverses, and the Si host atoms become positively charged while the $\mathrm{P}$ atom is negatively charged. ${ }^{1,2}$ Similar studies for Si surfaces doped with $\mathrm{P}$ or As atoms are rare and incomplete. They are important, however, as many nanoelectronic devices are designed to be formed on $\mathrm{Si}$ surfaces. ${ }^{3}$

Some studies of the interaction of $\mathrm{P}$ and As atoms with the technologically important $\mathrm{Si}(001)$ surface have been reported. Scanning tunneling microscopy (STM) experiments on a $\mathrm{Si}(001)$ surface exposed to arsenic have been carried out by Tromp et al. ${ }^{4}$ and Kipp et al. ${ }^{5}$ STM has also been em-

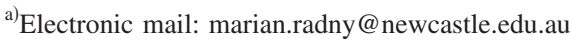

ployed to study P-related features on the $\mathrm{Si}(001)$ surface..$^{6-10}$ This experimental work, in conjunction with theory, ${ }^{11-13}$ has established that phosphorus atoms incorporate into the $\mathrm{Si}(001)$ surface to form $\mathrm{Si}-\mathrm{P}$ heterodimers where the $\mathrm{P}$ atom replaces a $\mathrm{Si}$ atom of a surface dimer. For $\mathrm{As}$ on $\mathrm{Si}(001)$, it has been shown that it is energetically favorable for As addimer atoms to exchange with two of the underlying $\mathrm{Si}$ atoms to create a Si-Si dimer on the surface. ${ }^{14}$ Dissociation of this $\mathrm{Si}-\mathrm{Si}$ dimer followed by diffusion results in the formation of $\mathrm{Si}-\mathrm{As}$ heterodimers on the surface. Our computational survey of possible products of $\mathrm{AsH}_{3}$ reacting with $\mathrm{Si}(001)$ suggests that the process of $\mathrm{AsH}_{3}$ chemisorption closely mirrors that of $\mathrm{PH}_{3},{ }^{15}$ further supporting the notion that $\mathrm{Si}-\mathrm{As}$ heterodimers are the end products of dissociation and substitutional incorporation.

Early STM studies of P-doped $\mathrm{Si}(001)$, in agreement with some theoretical predictions for $\mathrm{Si}-\mathrm{As}$ heterodimers ${ }^{14}$ and chemical intuition, ${ }^{6,7}$ concluded that the STM appearance of an isolated $\mathrm{Si}-\mathrm{P}$ heterodimer was consistent with a model in which the dangling bond of the heterodimer $\mathrm{Si}$ atom traps the donor electron. Recent bias-voltage dependent 
STM measurements, however, contradict these conclusions, finding instead that the donor electron is delocalized over the surface at low STM biases. ${ }^{13}$

Expanding on our previous brief report, ${ }^{13}$ we present in this paper the results of detailed density functional theory calculations of the structural and electronic effects associated with the $\mathrm{Si}-\mathrm{P}$ and $\mathrm{Si}-\mathrm{As}$ heterodimers that result from the incorporation of $\mathrm{P}$ and As dopant atoms into the $\mathrm{Si}(001)$ surface. In contrast to previous models, we show that pinning of the neighboring $\mathrm{Si}-\mathrm{Si}$ dimers and their different orientations with respect to the $\mathrm{Si}-\mathrm{P}$ heterodimer have pronounced effects on both the heterodimers and the doped surface. STM measurements for the $\mathrm{P}: \mathrm{Si}(001)$ system have revealed a complicated bias-voltage dependent STM appearance for an isolated $\mathrm{Si}-\mathrm{P}$ heterodimer on the electron rich $n$-type $\mathrm{Si}(001)$ surface $^{13}$ but not on the charge depleted $p$-type substrate. ${ }^{16}$ We show that these observations can be fully explained by progressive population of the unsaturated dangling bond of the heterodimer caused by STM tip-induced charge accumulation on $n$-type $\mathrm{Si}(001)$. An entirely similar behavior is predicted for the As:Si(001) system. It has also been shown that modeling an isolated heterodimer and its donor electron based on small atomic clusters ${ }^{6,7}$ or small unit cell periodic slab calculations ${ }^{14}$ may not be adequate to describe the complex electronic properties of surface dopant systems. The presented results are important as they can be readily generalized to many other adatom-dopant systems that lead to the formation of isolated, single dangling bond configurations on $\operatorname{Si}(001){ }^{17-19}$

\section{METHOD}

All geometry optimization and electronic structure calculations were carried out using the VASP plane-wave density functional method ${ }^{20-22}$ with a periodic surface slab model comprising $2 \times 4,2 \times 6,4 \times 4$, and $6 \times 6$ surface unit cells of either four or six Si layers. The bottom layer of each slab was terminated with a hydrogen layer and the vacuum region was $\sim 10 \AA$ thick. Brillouin zone integrations were performed using four special $\mathbf{k}$ points in the irreducible symmetry element of the surface Brillouin zone (SBZ). We carried out fully spin-polarized calculations using Vanderbilt ultrasoft pseudopotentials ${ }^{23}$ and the generalized gradient approximation for exchange and correlation (GGA-PW91). ${ }^{24}$ The surface electronic band structures were calculated using a periodic slab with a $4 \times 4$ surface unit cell and six Si layers by calculating the eigenenergies for each structure along the $\Gamma-J-K-J^{\prime}-\Gamma-K$ symmetry directions of the SBZ. In order to identify the nature of the surface states, three-dimensional charge/probability density distributions for all of the theoretically predicted surface states have been calculated. STM images of the Si-P heterodimer were simulated by invoking the Tersoff-Hamann approximation; ${ }^{25}$ the local density of states is integrated over a range of energy and converted into simulated constant-current STM images by tracing a specified isocontour. While this commonly used approximation will not yield an exact representation of the experimental STM images, it should reproduce all of the important qualitative features. We have checked that the features in the integrated (a)

(b)

(c)

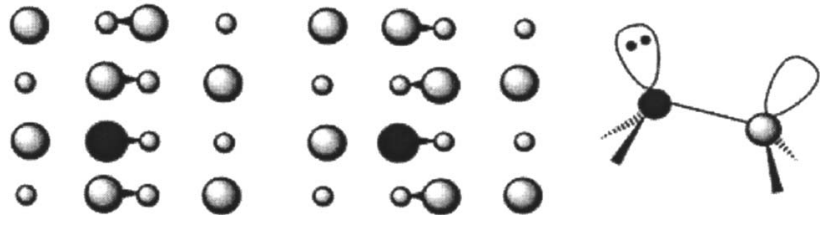

FIG. 1. Schematics of the (a) HD1 configuration, (b) HD2 configuration, and (c) buckled heterodimer structure on $\mathrm{Si}(001)$. The shaded small/large spheres represent the down/up-Si atoms of the buckled dimers, while the black spheres represent the P or As atom. Filled dots in (c) indicate the occupancy of the dangling bond by electrons.

local density of states (ILDOS) plots are relatively insensitive to the isosurface value and range of integration, provided all of the states important for imaging are included.

\section{RESULTS AND DISCUSSION}

\section{A. Ground state atomic structures}

Our calculations predict that for a single $\mathrm{P}$ or As atom incorporated into $\mathrm{Si}(001)$, there are two stable configurations independent of the size of the surface unit cell chosen. In one of these, the buckling angle of the heterodimer has the same sign as its two neighboring $\mathrm{Si}-\mathrm{Si}$ dimers in the same dimer row (HD1), while in the second configuration, the buckling angle has the opposite sign (HD2). These structures are shown schematically for the $4 \times 4$ surface unit cell in Figs. 1(a) and 1(b), respectively. These configurations are interchangeable by the inversion of the buckling angles of all of the $\mathrm{Si}-\mathrm{Si}$ dimers in the dimer row containing the $\mathrm{Si}-\mathrm{P}$ or $\mathrm{Si}-\mathrm{As}$ heterodimer. Our calculations also show that the heterodimer is always buckled with the dopant atom being the up atom [Fig. 1(c)]. This finding is in contrast to earlier semiempirical and molecular orbital cluster calculations which predicted that the Si-P geometry is a buckled dimer structure with the $\mathrm{P}$ atom being the down atom. ${ }^{26}$

\section{Si(001):P system}

We summarize in Table I our calculated bond lengths and buckling angles for the $\mathrm{Si}-\mathrm{P}$ heterodimer and adjacent $\mathrm{Si}-\mathrm{Si}$ dimers for the HD1 and HD2 configurations, focusing, in particular, on results obtained using different sized unit cells. For the HD2 configuration, the results are seen to be insensitive to cell size, exhibiting fairly consistent values of around $15.4^{\circ}$ and $2.30 \AA$ for the buckling angle and bond length of the Si-P heterodimer. Moreover, the bond lengths and buckling angles of the adjacent $\mathrm{Si}-\mathrm{Si}$ dimers are found to be quite similar to those of the clean $\mathrm{Si}(001)$ surface $\left(2.37 \AA\right.$ and $\left.18.9^{\circ}\right)$. By contrast, the structural parameters of the HD1 configuration are seen to be very sensitive to the choice of unit cell. The buckling angles and bond lengths of the $\mathrm{Si}-\mathrm{P}$ heterodimer change from $5.7^{\circ}$ and $2.31 \AA$ for the $2 \times 4$ cell to $15.1^{\circ}$ and $2.26 \AA$ for the $6 \times 6$ cell. The bond lengths and buckling angles of the adjacent $\mathrm{Si}-\mathrm{Si}$ dimers are similar to those calculated for the HD2 configuration and the free surface. This shows that the principal structural changes associated with the phosphorus atom in the $\mathrm{Si}(001)$ surface are localized to the $\mathrm{Si}-\mathrm{P}$ heterodimer. The energy difference 
TABLE I. Calculated dimer buckling angles (in degrees) and bond lengths (in $\AA$, given in parentheses) of the $\mathrm{Si}-\mathrm{P}$ heterodimer, and the nearest $(\mathrm{n})$ and next-nearest $(\mathrm{nn}) \mathrm{Si}-\mathrm{Si}$ dimers as a function of surface unit cell size.

\begin{tabular}{llcccc}
\hline \hline & Dimer & $2 \times 4$ & $2 \times 6$ & $4 \times 4$ & $6 \times 6$ \\
\hline HD1 & Si-P & $+5.7(2.31)$ & $+9.3(2.29)$ & $+14.6(2.26)$ & $+15.1(2.26)$ \\
& $\mathrm{Si}-\mathrm{Si}(\mathrm{n})$ & $+18.3(2.35)$ & $+18.3(2.36)$ & $+18.5(2.35)$ & $+18.5(2.34)$ \\
& $\mathrm{Si}-\mathrm{Si}(\mathrm{nn})$ & $-18.7(2.37)$ & $-18.9(2.37)$ & $-18.4(2.37)$ & $-18.6(2.37)$ \\
$\mathrm{HD} 2$ & $\mathrm{Si}-\mathrm{P}$ & $+15.6(2.30)$ & $+15.3(2.30)$ & $+15.5(2.31)$ & $+15.3(2.31)$ \\
& $\mathrm{Si}-\mathrm{Si}(\mathrm{n})$ & $-19.5(2.37)$ & $-19.2(2.37)$ & $-19.1(2.37)$ & $-18.9(2.37)$ \\
& $\mathrm{Si}-\mathrm{Si}(\mathrm{nn})$ & $+19.6(2.37)$ & $+19.3(2.37)$ & $+19.3(2.37)$ & $+18.9(2.37)$ \\
& & & & -0.14 & -0.14 \\
\hline (HD2) $-E(\mathrm{HD} 1)(\mathrm{eV})$ & -0.15 & -0.14 & & \\
\hline \hline
\end{tabular}

between the HD2 and HD1 configurations is calculated to be $\sim 0.14 \mathrm{eV}$ irrespective of the size of the surface unit cell, with the HD2 configuration being lower in energy. Observing from Table I that the structural parameters and energies obtained using the $4 \times 4$ and $6 \times 6$ unit cells are very similar, we conclude that the $4 \times 4$ cell provides a good description of an isolated heterodimer on $\mathrm{Si}(001)$. The remainder of the discussion will therefore be limited to $4 \times 4$ surface unit cells.

\section{Si(001): As system}

The bond lengths and bond angles characterizing the optimized geometries for the two configurations of an isolated $\mathrm{Si}-\mathrm{As}$ heterodimer are presented in Table II. In comparison with the $\mathrm{Si}-\mathrm{P}$ results included in Table II, we observe that the bond lengths and bond angles of the $\mathrm{Si}-\mathrm{Si}$ dimers adjacent to the $\mathrm{Si}-\mathrm{As}$ heterodimer are slightly larger (by $\sim 0.01 \AA$ and $0.8^{\circ}$ ) than those for the $\mathrm{Si}-\mathrm{P}$ heterodimer but still quite similar to the values of the clean surface. The bond length of the $\mathrm{Si}-\mathrm{As}$ heterodimer for each of the configurations is approximately $0.13 \AA$ longer than the corresponding $\mathrm{Si}-\mathrm{P}$ heterodimer bond length. The buckling angles of the $\mathrm{Si}-\mathrm{As}$ heterodimers are also around $3^{\circ}$ larger than those of their $\mathrm{Si}-\mathrm{P}$ counterparts. The HD2 configuration is predicted to be more stable than the HD1 configuration by $0.14 \mathrm{eV}$, exactly the same value as obtained for the $\mathrm{Si}-\mathrm{P}$ heterodimer. For comparison, we have also calculated the energies for $\mathrm{Si}-\mathrm{Si}$ dimers in similar buckling configurations as the heterodimers. The calculated energy difference was $0.09 \mathrm{eV}$, with the alternating buckling angle configuration again being the lower in energy (see Table II).

\section{B. Ground state electronic structures}

Both $\mathrm{Si}-\mathrm{P}$ and $\mathrm{Si}-\mathrm{As}$ heterodimers have one donor electron that is shared with the surface atoms. It has been predicted $^{6,7,14}$ that this electron would occupy the dangling bond orbital of isolated $\mathrm{Si}-\mathrm{P}$ and $\mathrm{Si}-\mathrm{As}$ heterodimers. We will show that this prediction is incorrect and that the different arrangements of the buckled $\mathrm{Si}-\mathrm{Si}$ dimers adjacent to the heterodimer for the HD1 and HD2 configurations result in two different electronic structures and quite different behaviors of the donor electron in the two cases.

\section{Si(001):P system}

The surface electronic structures calculated for the clean $\mathrm{Si}(001)$ surface and the HD1 and HD2 heterodimer configurations are shown in Figs. 2 and 3, respectively. In the HD2 band structure in Fig. 3(b), the levels associated with the P atom, including the doubly occupied dangling bond (lone pair), lie $\sim 2.0 \mathrm{eV}$ from the Fermi energy $\left(E_{F}\right)$ and hence are not present in the figure. As a result, the surface electronic structure of the HD2 configuration in the vicinity of $E_{F}$ is similar to that of the clean $\mathrm{Si}(001)$ surface (Fig. 2). A detailed band-structure population analysis for all of the electronic surface states in the vicinity of the Fermi energy reveals that there are two surface states that are significantly affected by the breaking of the $\mathrm{Si}-\mathrm{Si} \pi$ dimer bond. These states, which arise from the surface states that are intrinsic to the $\mathrm{Si}(001)$ surface, are denoted in the HD2 $\mathrm{Si}-\mathrm{P}$ band structure [Fig. 3(b)] by the open circles and filled squares. The surface state denoted by the open circles has a probability density that is predominantly localized on the heterodimer

TABLE II. Comparison of calculated buckling angles (in degrees) and bond lengths (in $\AA$, given in parentheses) for heterodimers $\mathrm{Si}-\mathrm{P}$ and $\mathrm{Si}-\mathrm{As}$. Values are also given for the $\mathrm{Si}-\mathrm{Si}$ dimers nearest (n) and next-nearest (nn) to the heterodimer, and for the clean $\operatorname{Si}(001)$ surface. All results were obtained using a $4 \times 4$ surface unit cell.

\begin{tabular}{llccc}
\hline \hline & Dimer & Si-P & Si-As & Si-Si \\
\hline HD1 & $\mathrm{Si}-X$ & $+14.6(2.26)$ & $+17.6(2.40)$ & $+15.9(2.31)$ \\
& $\mathrm{Si}-\mathrm{Si}(\mathrm{n})$ & $+18.5(2.35)$ & $+19.2(2.36)$ & $+18.2(2.34)$ \\
& $\mathrm{Si}-\mathrm{Si}(\mathrm{nn})$ & $-18.4(2.37)$ & $-19.2(2.38)$ & $-19.2(2.38)$ \\
$\mathrm{HD} 2$ & $\mathrm{Si}-X$ & $+15.5(2.31)$ & $+18.4(2.44)$ & $+18.9(2.37)$ \\
& $\mathrm{Si}-\mathrm{Si}(\mathrm{n})$ & $-19.1(2.37)$ & $-19.9(2.38)$ & $-18.9(2.37)$ \\
& $\mathrm{Si}-\mathrm{Si}(\mathrm{nn})$ & $+19.3(2.37)$ & $+20.0(2.38)$ & $+18.9(2.37)$ \\
& & & -0.14 & -0.09 \\
\hline \hline
\end{tabular}




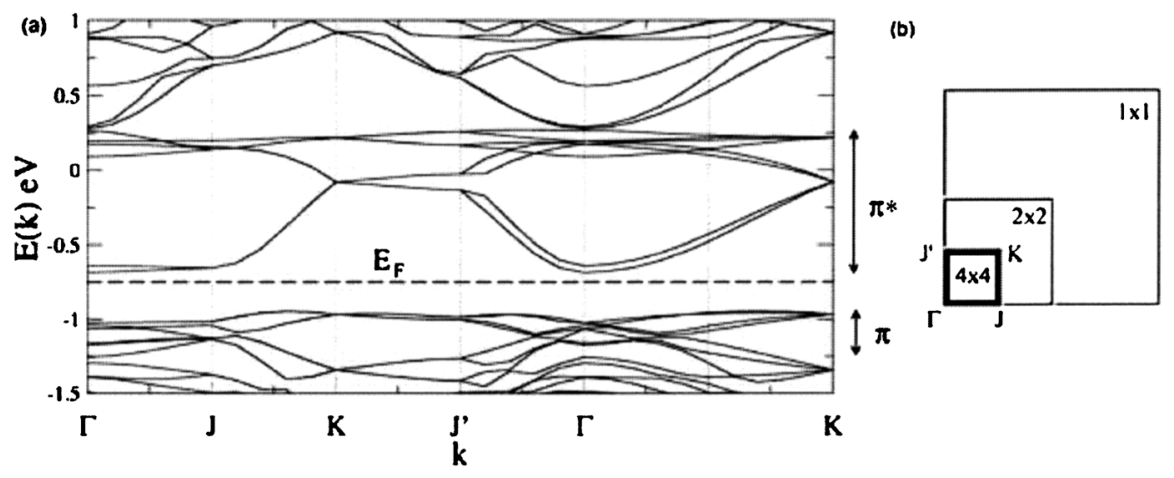

FIG. 2. (a) The surface electronic structure of the clean $\operatorname{Si}(001)$ surface calculated using a $4 \times 4$ surface unit cell with six Si layers in the vicinity of the Fermi level $E_{F}$ (indicated by the dashed horizontal line). The occupied $(\pi)$ and unoccupied $\left(\pi^{*}\right)$ bands of electronic surface states are shown; (b) the irreducible symmetry element of the $4 \times 4$ SBZ.

down-Si atom and therefore represents the unoccupied heterodimer Si atom dangling bond orbital. The probability density of this state is shown in Fig. 4(a). The energy of this state lies well above $E_{F}$ among other localized (relatively flat) surface state bands that form the upper part of the unoccupied $\pi^{*}$ band. The dispersive, partially occupied surface state denoted by the filled squares also has the maximum of its charge/probability density localized on the down-Si atom of the heterodimer, as well as contributions from all of the other down-Si dimer atoms in the same dimer row [see Fig. 4(b)]. As the donor electron only occupies this dispersive band, it is effectively delocalized on the surface and not present in the Si dangling bond.

The surface electronic structure calculated for the HD1 configuration is shown in Fig. 3(a). In contrast to the HD2 configuration, the band structure for HD1 exhibits a well pronounced surface state just above the Fermi level within the unoccupied $\pi^{*}$ band (denoted by the open circles). This state has a probability density that is strongly localized on the down-Si atom of the heterodimer and represents the unoccupied heterodimer $\mathrm{Si}$ atom dangling bond orbital. This state is largely decoupled from the $\pi^{*}$ surface bands by the breaking of the Si-Si dimer $\pi$ bond, and has the same origin as the state in the electronic structure of the HD2 configuration denoted by the open circles in Fig. 3(b). Since the buckling angles of both the HD1 and HD2 heterodimer configurations are nearly identical, the downward shift in energy of the heterodimer $\mathrm{Si}$ atom dangling bond state for the HD1

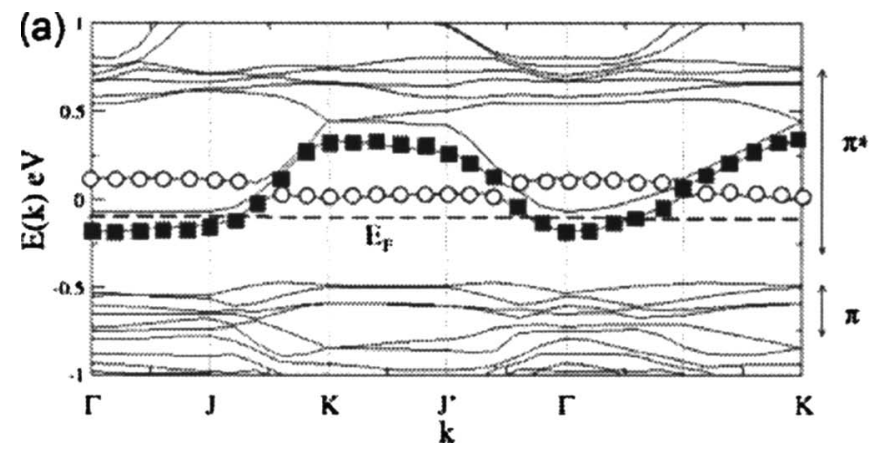

structure must be a consequence of the disrupted periodicity within the $\mathrm{Si}-\mathrm{Si}$ dimer row of this structure. This disrupted periodicity is due to the formation of a three-dimer wide defect comprising the heterodimer and its two neighboring $\mathrm{Si}-\mathrm{Si}$ dimers, all of which are buckled in the same direction [see Fig. 1(a)]. The dispersive, partially occupied surface state denoted by the filled squares in Fig. 3(a) describes the donor electron delocalized on the surface. This state is similar to that shown in Fig. 3(b) for the HD2 configuration but, as we shall see below, has most of its charge/probability density localized on the down-Si atoms of the dimer row not containing the heterodimer.

\section{Si(001):As system}

The electronic band structures for the optimized HD1 and HD2 Si-As heterodimer structures are presented in Figs. 5(a) and 5(b), respectively. These band structures are very similar to those presented in Fig. 3 for the P:Si(001) system. As for the Si-P heterodimer, there are two surface states that are substantially affected by the breaking of the $\mathrm{Si}-\mathrm{Si}$ dimer $\pi$ bond. These states are indicated in Fig. 5 by the filled squares and empty circles and exhibit exactly the same behavior and properties as those described for the HD1 and HD2 configurations of the Si-P heterodimer. Again, the donor electron only occupies the dispersive state denoted by the

FIG. 3. The surface electronic structure of the (a) HD1 and (b) HD2 Si-P configurations calculated with a six layer $4 \times 4$ surface unit cell slab, with the heterodimer Si dangling bond surface states denoted by symbols (see text). The dashed lines represent the Fermi level. The bands corresponding to the minority and majority spin electrons are identical. 


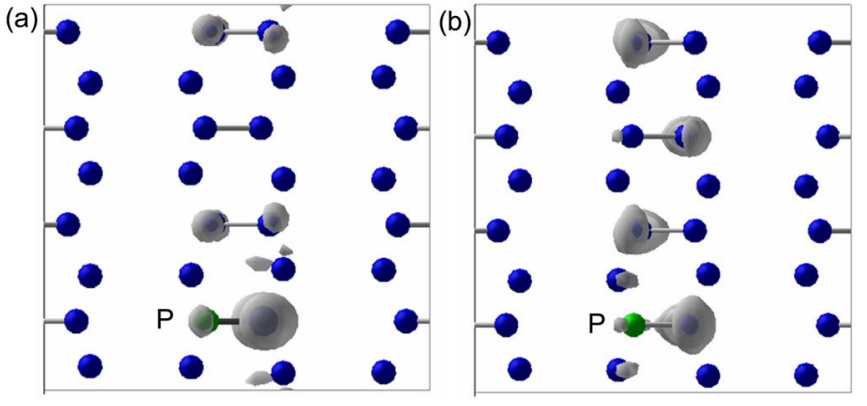

FIG. 4. (Color online) Top views of the charge/probability densities of the (a) heterodimer Si dangling bond orbital [open circles in Fig. 3(b)] and (b) partially occupied state crossing the Fermi energy [filled squares in Fig. $3(b)]$ for the HD2 heterodimer configuration and the $\Gamma$ point of the SBZ. The plots are for an isosurface value of $1.4 \times 10^{-2} e \AA^{-3}$. Only atoms from the first and second layers are shown, and the dimer bonds are indicated by the solid lines.

filled squares in Fig. 5. We would therefore expect the donor electron to be delocalized over the surface for both the HD1 and HD2 configurations.

\section{The unpaired (donor) electron}

We have shown that on the $\mathrm{Si}(001)$ surface in the HD1 and HD2 configurations for both the P:Si and As:Si systems, the donor electron is delocalized over the surface. In an infinite system, an impurity (or dopant) such as phosphorus or arsenic produces a "shallow" state within the energy gap. Such a state is a result of the long range Coulombic potential that arises because of the different number of electrons on the dopant atom. On the doped $\mathrm{Si}(001)$ surface, however, the donor electron for both the HD1 and HD2 surface configurations occupies the lowest energy surface state within the unoccupied $\pi^{*}$ band. As such, this state is not strictly a defect level but is intrinsic to the clean $\operatorname{Si}(001)$ surface. It is only the occupancy of this state that changes because of the presence of the donor electron on the surface. The unoccupied surface states that constitute the $\pi^{*}$ band originate predominantly from the $p_{z}$-like empty dangling bond orbitals on the Si-down atoms of the buckled $\mathrm{Si}-\mathrm{Si}$ dimers. These states lie in the Si bulk energy gap with a negligible mix with the bulk states. The delocalized charge populating these states is thus spread laterally on the surface instead of traveling to the bulk states.

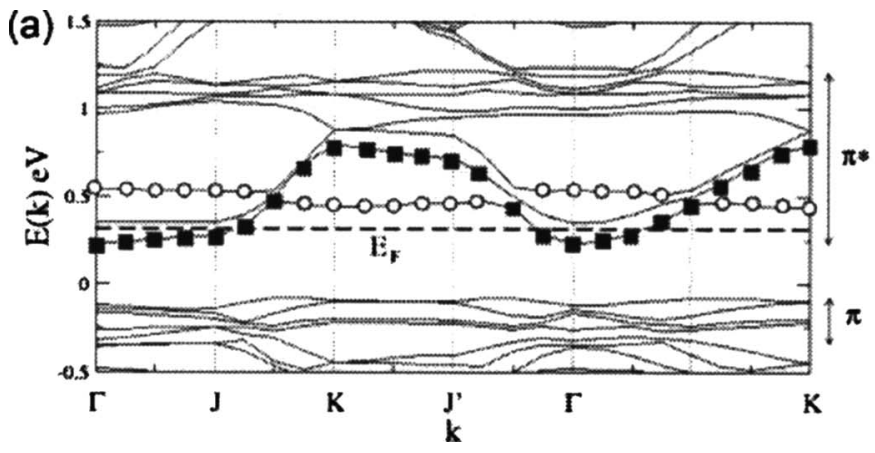

(a)

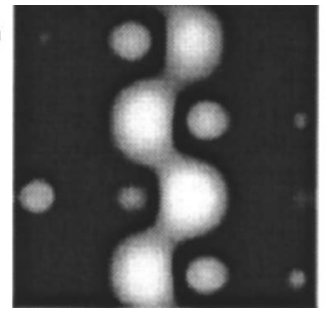

(b)

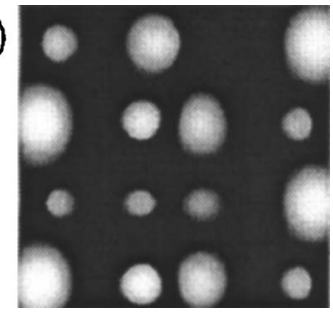

FIG. 6. Filled-state ILDOS plots obtained by integrating over $-0.2 \mathrm{eV}$ from the Fermi energy for the $4 \times 4$ surface unit cell (a) HD2 and (b) HD1 configurations. The plots are for the isosurface value of $2.7 \times 10^{-4} e / \AA^{-3}$.

The partially occupied state has a very similar dispersion along the high symmetry directions of the SBZ for both the HD1 and HD2 configurations. As can be seen in Figs. 3 and 5 , this state is dispersive along the $J-K$ and $J^{\prime}-\Gamma$ symmetry directions but exhibits negligible dispersion in the $\Gamma-J$ and $K-J^{\prime}$ symmetry directions. This indicates that the donor electron is localized in the direction perpendicular to the $\mathrm{Si}-\mathrm{P} / \mathrm{Si}-\mathrm{As}$ dimer row but delocalized along the dimer rows. The spatial distribution of the delocalized charge for the $\mathrm{Si}-\mathrm{P}$ heterodimer in the HD2 configuration is shown in Fig. 6(a). This plot has been obtained by integrating the local density of states over $-0.2 \mathrm{eV}$ from $E_{F}$ and hence shows the spatial distribution of the charge associated with just the half-filled surface state. The donor electron is seen to be localized in the direction perpendicular to the dimer rows but delocalized over the surface along the dimer row containing the Si-P heterodimer. The analogous ILDOS plot for the HD1 configuration is shown in Fig. 6(b). In this case, the donor electron is delocalized predominantly over the $\mathrm{Si}-\mathrm{Si}$ dimer rows adjacent to the row containing the Si-P heterodimer. This quite distinct behavior is caused by the different orientations of the $\mathrm{Si}-\mathrm{Si}$ dimers adjacent to the heterodimer in the HD1 and HD2 configurations, and the formation of the three-dimer wide surface defect in the case of HD1.

Since a delocalized charge does not change the bonding structure of a covalent system, the different characters of the delocalization of the donor electron for the two configurations will not affect the geometry of the heterodimers. This explains why the heterodimer buckling angles and bond lengths are virtually the same for the HD1 and HD2 configurations. Moreover, the delocalized donor electron renders the

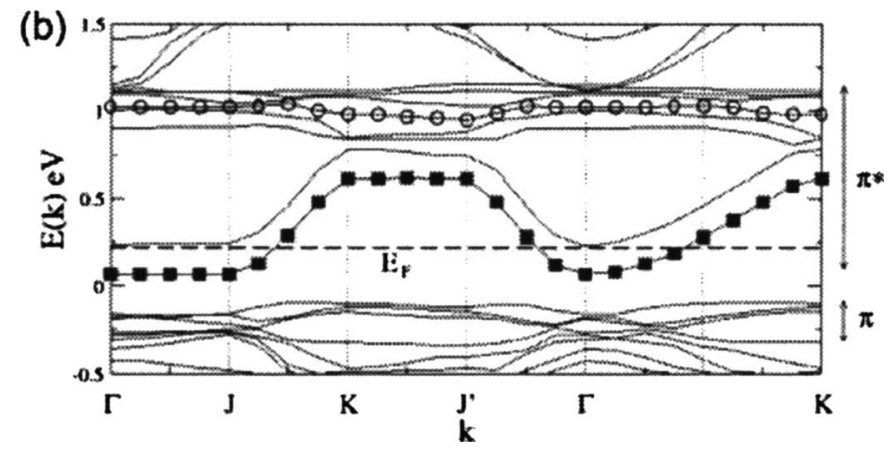

FIG. 5. The surface electronic structure of the (a) HD1 and (b) HD2 Si-As configurations calculated with a six layer, $4 \times 4$ surface unit cell slab, with the heterodimer Si dangling bond surface states denoted by symbols (see text). The dashed lines represent the Fermi level. The bands corresponding to the minority and majority spin electrons are identical. 
TABLE III. Si-P and $\mathrm{Si}-\mathrm{As}$ heterodimer buckling angles (in degrees) and bond lengths (in $\AA$, given in parentheses) calculated for the HD1 and HD2 configurations using a $4 \times 4$ surface unit cell.

\begin{tabular}{|c|c|c|c|c|c|}
\hline & & $N_{e}-1$ & $N_{e}$ & $N_{e}+1$ & $N_{e}+2$ \\
\hline \multirow[t]{2}{*}{ HD1 } & $\mathrm{Si}-\mathrm{P}$ & $+15.9(2.25)$ & $+14.6(2.26)$ & $+3.2(2.33)$ & $-5.7(2.42)$ \\
\hline & $\mathrm{Si}-\mathrm{As}$ & & $+17.6(2.40)$ & $+6.4(2.46)$ & $-2.5(2.56)$ \\
\hline \multirow[t]{2}{*}{ HD2 } & $\mathrm{Si}-\mathrm{P}$ & $+15.4(2.31)$ & $+15.5(2.30)$ & $+15.7(2.30)$ & $+15.8(2.29)$ \\
\hline & $\mathrm{Si}-\mathrm{As}$ & & $+18.4(2.44)$ & $+18.3(2.43)$ & $+18.0(2.42)$ \\
\hline
\end{tabular}

heterodimer locally cationic, and therefore locally isoelectronic to a $\mathrm{Si}-\mathrm{Si}$ dimer on the clean $\mathrm{Si}(001)$ surface. It is thus not surprising that the predicted buckling angles for the $\mathrm{Si}-\mathrm{P}$ $\left(\sim 15^{\circ}\right)$ and $\mathrm{Si}-\mathrm{As}\left(\sim 18^{\circ}\right)$ heterodimers are quite similar to those of a $\mathrm{Si}-\mathrm{Si}$ dimer on the clean $\mathrm{Si}-\mathrm{Si}$ surface (see Table II). As has been shown, this similarity also extends to the electronic structure and the energetics. The HD2 structure has been found to be $\sim 0.14 \mathrm{eV}$ more stable than the HD1 configuration for both the $\mathrm{Si}-\mathrm{P}$ and $\mathrm{Si}-\mathrm{As}$ heterodimers (see Table II). This value is consistent with the value of $0.13 \mathrm{eV}$ obtained for the corresponding structures of $\mathrm{Si}-\mathrm{Si}$ dimers on the clean $\mathrm{Si}(001)$ surface by Chen and Boland ${ }^{27}$ and our calculated energy difference of $0.09 \mathrm{eV}$ (see Table II).

\section{Effect of surface charge accumulation}

As discussed in Sec. III B, the donor electron occupies the lowest energy surface state within the $\pi^{*}$ band and is delocalized over the surface. A higher heterodimer coverage would increase the density of donor electrons on the surface leading to an effective surface charge accumulation. A higher heterodimer coverage in experiment corresponds to a reduction in the size of the surface unit cell in our calculations. Our calculations predict that the buckling angle and bond length of the $\mathrm{Si}-\mathrm{P}$ heterodimer in the $\mathrm{HD} 1$ configuration change from $5.7^{\circ}$ and $2.31 \AA$ for the $2 \times 4$ unit cell to $14.6^{\circ}$ and $2.26 \AA$ for the $4 \times 4$ unit cell (see Table I). These changes in buckling angle and bond length indicate charging of the $\mathrm{Si}-\mathrm{P}$ heterodimer and suggest that it is the increase in the density of electrons on the surface that forces them to localize on the heterodimer Si site. In this section we investigate the effect of surface electron accumulation on the HD1 and $\mathrm{HD} 2 \mathrm{Si}-\mathrm{P}$ and $\mathrm{Si}-\mathrm{As}$ heterodimer configurations. In order to do this, we have carried out geometry optimization and electronic structure calculations for both configurations using a $4 \times 4$ surface unit cell containing $N_{e}-1, N_{e}+1$, and $N_{e}+2$ electrons (where $N_{e}$ is the number of electrons in the neutral system). The calculations were performed with a uniform background compensating charge. Tests were also carried out to ensure that the geometries and electronic structures obtained from these charged surface unit cell calculations are meaningful (see the Appendix).

\section{Si(001): P system}

The calculated buckling angles and bond lengths of the progressively charged heterodimers are presented in Table III. As can be seen, the HD2 configuration remains virtually unaffected by the addition of electrons to the system, while the HD1 configuration progressively changes from a buckled heterodimer $\left(\sim 15^{\circ}\right)$ in the $N_{e}-1$ and $N_{e}$ cases, via a near planar heterodimer $\left(\sim 3^{\circ}\right)$ in the $N_{e}+1$ case, to an upward buckling of the heterodimer $\mathrm{Si}$ atom $\left(\sim-6^{\circ}\right)$ for the $N_{e}+2$ case. The heterodimer bond length also increases from $2.25 \AA\left(N_{e}-1\right)$ to $2.42 \AA\left(N_{e}+2\right)$. These data indicate progressive charge transfer to the heterodimer $\mathrm{Si}$ atom in the HD1 configuration, but not in the HD2 configuration. The calculated surface electronic band structures support this prediction.

The electronic structures of the $N_{e}-1$ configurations are very similar to the corresponding $N_{e}$ band structures as there is little change in the respective geometries of the $N_{e}-1$ and $N_{e}$ configurations. This is not true, however, for the electronic band structures of the $N_{e}+1$ and $N_{e}+2$ Si-P HD1 configurations in the vicinity of $E_{F}$ (see Fig. 7). All of the identified surface state bands have virtually identical charge/ probability distributions as the corresponding bands of the neutral $N_{e}$ HD1 band structure shown in Fig. 3(a). Progres-
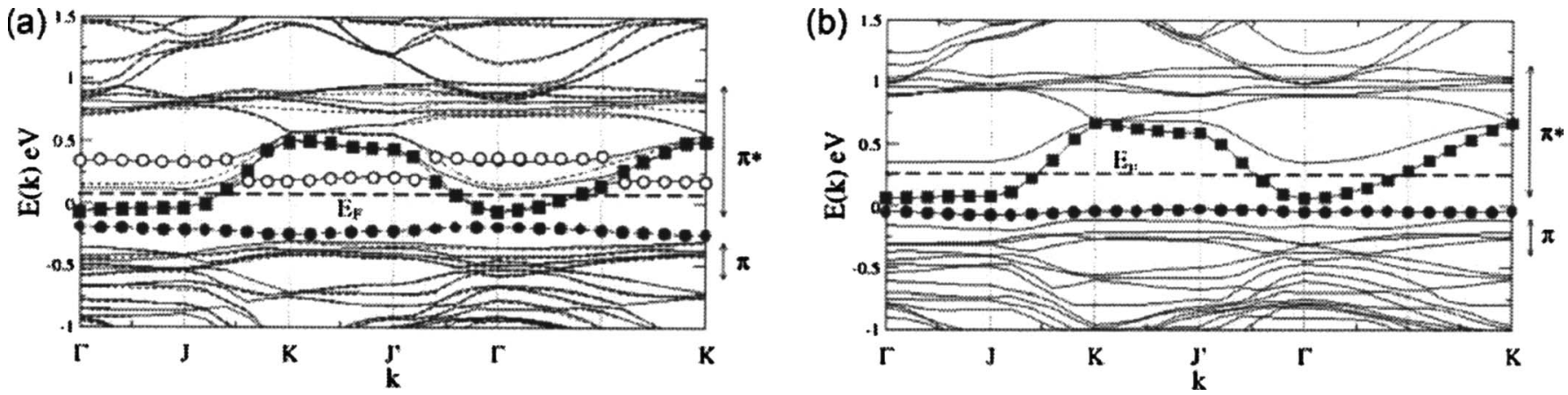

FIG. 7. The surface electronic structures of the charged HD1 (a) $N_{e}+1$ and (b) $N_{e}+2 \mathrm{Si}-\mathrm{P}$ configurations for the $4 \times 4$ surface unit cell and six Si layers. The dashed lines represent the Fermi level. The solid lines denote the minority spin energy bands, and the dotted lines indicate the bands for the majority spin electrons. 
sive charging of the system, however, induces significant changes to the energies of the localized heterodimer dangling bond levels. For the $N_{e}+1$ HD1 configuration with one additional electron [Fig. 7(a)], the two different spin states associated with the unoccupied dangling bond surface state band indicated by the empty circles in Fig. 3(a) become decoupled and split, with one state [filled circles in Fig. 7(a)] lying below $E_{F}$ and the other (empty circles) above $E_{F}$. This behavior is typical of an isolated dangling bond and is strongly influenced by electron correlation effects similar to those known for other isolated dangling bond systems. ${ }^{28,29}$ Both of these states have their charge/probability density strongly localized on the down-Si atom of the heterodimer. For the charged HD1 configuration with two additional electrons [Fig. 7(b)], both of these spin states are pushed down in energy below $E_{F}$ and become doubly occupied (filled circles). It is important to notice that when charging occurs, this localized state shifts into the band gap between the top of the valence band and the Fermi level [Fig. 7(b)]. This removes any coupling to the $\pi^{*}$ band and leads to fully localized dangling bond electrons. For both the HD1 $N_{e}+1$ and $N_{e}+2$ charged configurations, the partially occupied surface state that crosses the Fermi level has a spatial charge distribution that is very similar to that shown in Fig. 6(b) for the neutral HD1 system; that is, the electrons that remain in the lower part of the $\pi^{*}$ surface band are delocalized on the $\mathrm{Si}-\mathrm{Si}$ dimers of the neighboring dimer rows.

For the $N_{e}+1$ and $N_{e}+2 \mathrm{HD} 2$ configurations, the surface electronic band structures for the minority and majority spin electrons are identical and are essentially the same as that shown in Fig. 3(b) for the neutral HD2 configuration. The only noticeable differences are an upward shift of the Fermi level, a corresponding downward shift of the partially occupied surface state associated with the $\mathrm{Si}-\mathrm{Si}$ dimer row containing the Si-P heterodimer [see Fig. 4(b)], and a broadening of the $\pi^{*}$ surface band. As a result, any added electrons simply occupy the dispersive states of the $\pi^{*}$ surface band and hence remain delocalized on the surface.

The predicted electronic transitions and associated structural changes of the $\mathrm{Si}-\mathrm{P}$ heterodimer in the HD1 configuration are in excellent agreement with the experimental voltage-dependent STM images. ${ }^{13}$ In STM on $n$-type doped $\mathrm{Si}(001)$, the negative bias voltage pushes the electronic bands downward resulting in surface charge accumulation and population of the low energy unoccupied surface states within the $\pi^{*}$ band. ${ }^{30-32,13}$ To simulate the effects of STM tip-induced charge accumulation on the $n$-type substrate with increasing negative bias voltage, we have presented in Figs. 8(a)-8(c) images for the HD1 configuration corresponding to zero $\left(N_{e}\right)$, one $\left(N_{e}+1\right)$, and two $\left(N_{e}+2\right)$ additional electrons, respectively. The corresponding experimental STM filledstate images are also shown in Fig. 8 for comparison.

At low negative bias voltage, the low density of accumulated charge leaves the electrons delocalized and the heterodimer Si atom dangling bond unoccupied. This results in the dark, low-bias filled-state STM appearance of the heterodimer Si atom $\left[N_{e}\right.$ case, Fig. 8(a)]. In contrast, at high negative bias, the density of the accumulated electrons in the $\pi^{*}$ band increases, and two electrons are localized on the
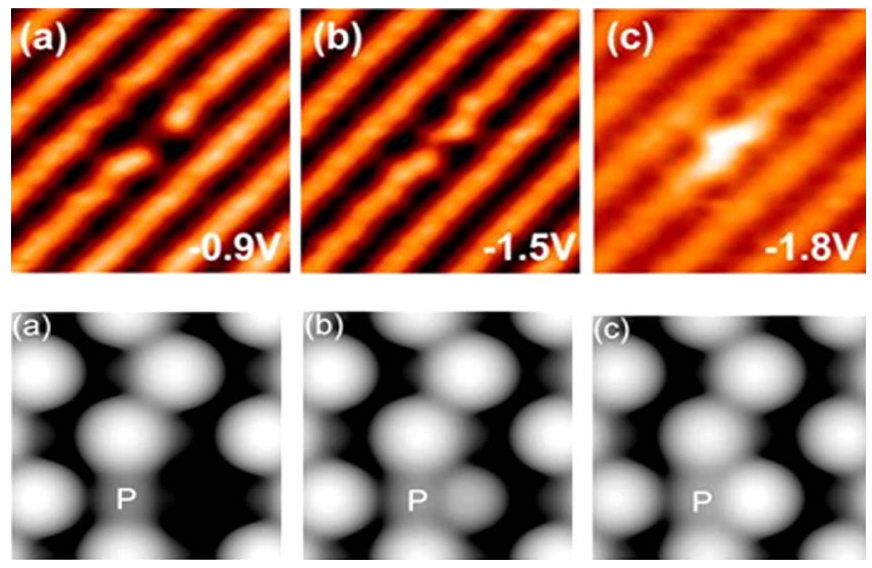

FIG. 8. (Color online) The experimental (top) and simulated (bottom) filledstate STM images of a Si-P heterodimer on the $n$-type $\mathrm{Si}(001)$ surface. The simulated images correspond to integrating over $1.0 \mathrm{eV}$ from the Fermi energy and are for the (a) $N_{e}$, (b) $N_{e}+1$, and (c) $N_{e}+2$ HD1 configurations. The experimental image at low bias (a) shows a Si-P heterodimer in the central region of the image as a relatively dark feature compared to the buckled atoms of the neighboring pinned $\mathrm{Si}-\mathrm{Si}$ dimers. With increased bias, this feature becomes brighter (b), before adopting the bright buckled appearance at high bias (c).

heterodimer Si atom dangling bond, shifting the heterodimer dangling bond level below $E_{F}$. The corresponding STM image in Fig. 8(c) shows the heterodimer Si atom to now be brighter than its neighboring up-buckled $\mathrm{Si}$ atoms. The fact that the $\mathrm{Si}-\mathrm{P}$ heterodimer and its immediately adjacent $\mathrm{Si}-\mathrm{Si}$ dimers are not as bright as they appear in the experimental image [see Fig. 8(c)] is most likely due to the neglect of any energy-dependent tunneling effects in the Tersoff-Hamann approximation. The charging of the heterodimer occurs via occupation of the dangling bond states close to $E_{F}$. These states have a smaller barrier through which to tunnel than states deeper in the valence band, and hence would be enhanced by the inclusion of such effects.

For the transitional $N_{e}+1$ state, when just one electron occupies the dangling bond of the heterodimer $\mathrm{Si}$ atom, the two different spin states split into two bands, one below $E_{F}$ and one above [see Fig. 7(a)], resulting in the medium-bias filled-state STM appearance shown in Fig. 8(b). In equivalent experiments on the $p$-type doped $\operatorname{Si}(001)$ surface, ${ }^{16}$ depletion of the surface electronic charge occurs due to downward band bending and the heterodimer $\mathrm{Si}$ atom dangling bond level remains above the Fermi level for any negative bias voltage. The heterodimer $\mathrm{Si}$ atom dangling bond is therefore always unoccupied, resulting in the dark appearance of the heterodimer $\mathrm{Si}$ atom for all negative bias voltages, identical to the image shown in Fig. 8(a). It is important to stress that this excellent agreement between theory and experiment can only be obtained for the HD1 configuration. The dangling bond surface state associated with the $\mathrm{Si}$ atom of the Si-P heterodimer in the HD2 configuration lies too high above the Fermi energy to be populated by electron accumulation on the surface. As a result, the $\mathrm{Si}-\mathrm{P}$ heterodimer will remain dark in filled state at all biases. It thus follows that the HD2 configuration cannot reproduce the negative bias dependence evidenced by the STM topographs in Figs. 8(a)-8(c). 

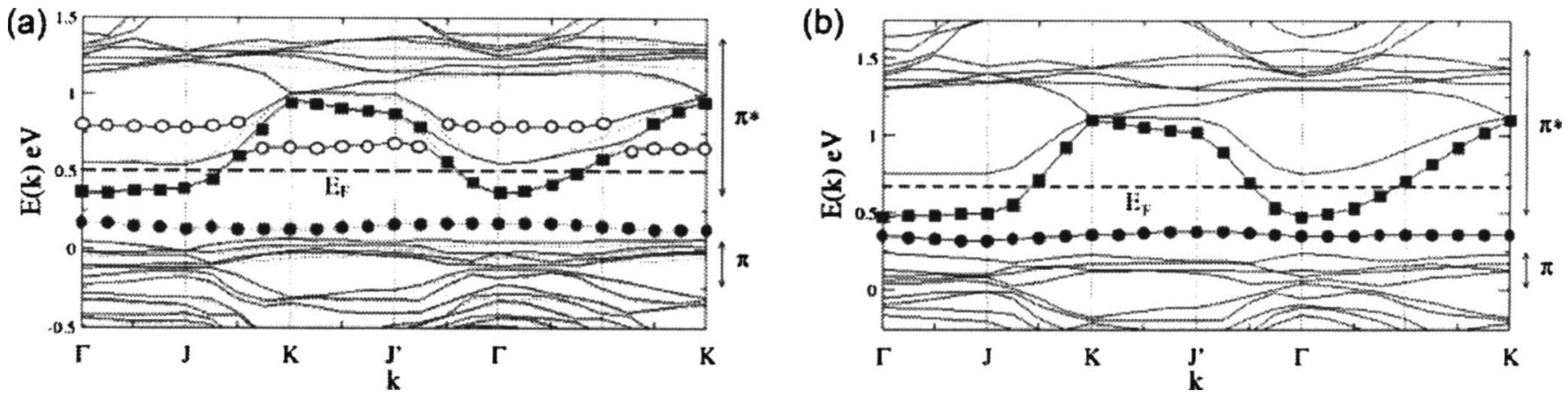

FIG. 9. The surface electronic structures of the charged HD1 (a) $N_{e}+1$ and (b) $N_{e}+2 \mathrm{Si}-$ As configurations for the $4 \times 4$ surface unit cell and six Si layers. The dashed lines represent the Fermi level. The solid lines denote the minority spin energy bands, and the dotted lines indicate the bands for the majority spin electrons.

\section{Si(001): As system}

The results of our geometry optimization calculations for the As: $\mathrm{Si}(001)$ system with one and two extra electrons (i.e., for the $N_{e}+1$ and $N_{e}+2$ systems) are also given in Table III. The obtained data mirror those for the Si-P heterodimers. The values for the HD2 configuration are observed to be changed little by progressive charging of the system, while the HD1 heterodimer buckling angle changes dramatically from $17.6^{\circ}$ for the neutral $N_{e}$ case to $6.4^{\circ}$ for the $N_{e}+1$ case and then to $-2.5^{\circ}$ for the $N_{e}+2$ configuration. The overall change in the bond length of the HD1 configuration of $0.16 \AA$ in going from the $N_{e}$ to the $N_{e}+2 \mathrm{Si}-\mathrm{As}$ heterodimer configuration is identical to the corresponding change for the $\mathrm{Si}-\mathrm{P}$ heterodimer. The overall changes in the buckling angles are also very similar with the values of $20.1^{\circ}$ and $20.3^{\circ}$, respectively.

The changes in both the bond length and buckling angle of the $\mathrm{Si}-\mathrm{As}$ heterodimer for the HD1 configuration imply that charge is being transferred to the heterodimer $\mathrm{Si}$ atom which will change the energy distribution of levels associated with this defect site. This is shown in the electronic band structures for the $N_{e}+1$ and $N_{e}+2$ HD1 configurations presented in Fig. 9. We observe that the two degenerate spin states of the unoccupied dangling bond surface state band for the neutral system indicated by the empty circles in Fig. 5(a) split apart in energy for the $N_{e}+1$ system so that one state lies above $E_{F}$ and the other below $E_{F}$ [Fig. 9(a)]. For the $N_{e}+2$ configuration, one obtains a single doubly degenerate level below $E_{F}$ which is completely decoupled from the other states [Fig. 9(b)]. The electronic band structures for the $N_{e}$ +1 and $N_{e}+2$ HD2 systems, on the other hand, are essentially the same as that shown in Fig. 5(b) for the neutral $N_{e}$ system apart from an upward shift of the Fermi level. These results are entirely analogous to those of the $\mathrm{Si}-\mathrm{P}$ heterodimer, and hence we would expect the $\mathrm{Si}-\mathrm{As}$ heterodimer to exhibit a very similar bias-voltage dependence in STM experiments.

\section{SUMMARY AND CONCLUSIONS}

We have carried out first-principles density functional theory (DFT) calculations to account for the structural and electronic properties of isolated $\mathrm{P}$ and As dopant atoms which form $\mathrm{Si}-\mathrm{P}$ and $\mathrm{Si}-\mathrm{As}$ heterodimer structures on the
$\mathrm{Si}(001)$ surface. Calculations were performed for both the neutral and charged P:Si(001) and As:Si(001) substitutional systems.

From our ground state calculations for the neutral $N_{e}$ system, we have shown that the formation of an isolated $\mathrm{Si}-\mathrm{P}$ or $\mathrm{Si}-\mathrm{As}$ heterodimer on $\mathrm{Si}(001)$ leads to two stable configurations. The electronic structures for the charged HD1 configurations have been shown to exhibit splitting resulting from strong electron correlation effects that are characteristic of an isolated dangling bond. The calculations have also revealed that it is the appearance of the unoccupied electronic state associated with the heterodimer $\mathrm{Si}$ atom dangling bond near the Fermi energy that makes the HD1 configuration susceptible to the electronic and structural instabilities induced by surface charge accumulation. By contrast, the HD2 configuration is not significantly affected by the accumulation of electrons on the surface.

In previous calculations, it has been assumed that the local atomic reconstruction of the heterodimer is responsible for the main features of the system observed in STM and that the presence of the $\mathrm{Si}-\mathrm{Si}$ dimers around the $\mathrm{Si}-\mathrm{P}$ heterodimer is not important. ${ }^{4,5}$ The calculations presented here clearly demonstrate that while the heterodimer buckling angles on the P-incorporated and As-incorporated $\mathrm{Si}(001)$ surfaces are very similar for the neutral HD1 and HD2 configurations, the different $\mathrm{Si}-\mathrm{Si}$ dimer configurations around an isolated heterodimer lead to quite different behaviors for both the heterodimer and the doped surface. Moreover, we have shown that doping $\mathrm{Si}(001)$ via the substitutional incorporation of dopant atoms leads to the formation of an extended surface defect which must be modeled with great care.

The DFT energies that we have determined for isolated $\mathrm{Si}-\mathrm{P}$ and $\mathrm{Si}-\mathrm{As}$ heterodimers for the neutral (uncharged) systems show a clear energy preference for the HD2 configuration. We have demonstrated, however, that the experimentally observed $\mathrm{Si}-\mathrm{P}$ heterodimer features originate from the HD1 configuration, and that only the electronic and structural phenomena associated with this configuration are consistent with the experimental data. ${ }^{13}$ Intuitively, one would expect the heterodimer and its neighboring $\mathrm{Si}-\mathrm{Si}$ dimers to form an antiparallel buckling configuration to minimize the surface strain, consistent with our prediction of HD2 as the lower energy structure. Under experimental bias-voltage conditions, tip-induced band bending leads to either a deple- 
tion or accumulation of charge on the surface. For large negative bias, our calculations predict that charge accumulation results in the parallel buckling HD1 configuration being transformed into an antiparallel buckling sequence, consistent with our intuition that such a configuration would yield a lower energy. However, for other voltages, a parallel dimer buckling sequence is observed. To understand why the HD1 configuration is the preferred structure for these bias voltages, we really need to consider charged systems. Unfortunately, we cannot obtain accurate energies for the HD1 and HD2 charged systems from the VASP code, and hence a direct comparison of the relative energies of these two configurations for differing degrees of surface charging is not possible. Resolution of why the HD1 structure is observed in the experiments must therefore await the development of a reliable theoretical method for determining the energetics of charged semiconductor:adsorbate systems.

High resolution bias-dependent STM measurements for the As: $\mathrm{Si}(001)$ system are required to discover whether the same behavior is characteristic of $\mathrm{Si}-\mathrm{As}$ heterodimers on $\mathrm{Si}(001)$. The similarity in behavior of the $\mathrm{Si}-\mathrm{As}$ and $\mathrm{Si}-\mathrm{P}$ heterodimers evidenced in this paper, however, suggests that this is indeed likely to be the case.

\section{ACKNOWLEDGMENTS}

This work was supported by the Australian Research Council, the Australian Government, the Semiconductor Research Corporation, the US Advanced Research and Development Activity, the National Security Agency, and the Army Research Office under Contract No. DAAD19-01-1-0653. We also want to acknowledge the Australian Partnership for Advanced Computing for an award under the Merit Allocation Scheme and I. Itagaroulias for performing some of the $\mathrm{Si}-\mathrm{As}$ heterodimer calculations.

\section{APPENDIX: CONVERGENCE TESTS FOR CHARGED CELLS}

A possible source of error in our calculations for charged systems may arise from interactions between neighboring cells resulting from the periodic boundary conditions. These interactions should be most significant when each cell has a large asymmetrical charge distribution (and hence a large dipole/quadrupole moment). In order to test for such effects, we first carried out calculations progressively increasing the slab height (i.e., the thickness of the vacuum layer) from 10.0 to $25.0 \AA$ while retaining the $4 \times 4$ surface unit cell in the $x y$ plane. Calculations were performed for both the HD1 and HD2 Si-P configurations for the $N_{e}-1, N_{e}+1$, and $N_{e}$ +2 charged systems. In all cases, the optimized geometries remained essentially invariant, and the total energies and their associated energy differences were found to exhibit convergent behavior. We can thus conclude that any unwanted interactions in the $z$ direction due to the periodic boundary conditions must be negligible.
To test whether this was also true in the lateral $(x y)$ directions, we performed geometry optimization calculations for the HD1 $N_{e}+1$, HD2 $N_{e}+1$, and HD1 $N_{e}+2$ Si-P heterodimer systems for both $4 \times 4$ and $6 \times 6$ surface unit cells. All of these calculations were performed using just $\Gamma$-point sampling and varying only the atoms in the dimer row containing the $\mathrm{Si}-\mathrm{P}$ heterodimer. The optimized geometries obtained from these $6 \times 6$ surface unit cell calculations were found to be virtually identical to those obtained from the 4 $\times 4$ calculations. This confirms that there are no significant structural variations arising from the imposition of periodic boundary conditions in the xy plane.

Taken together with the earlier tests of the $z$ dependence, these results enable us to conclude that our predicted geometries (and hence the corresponding electronic structures) for these charged systems should be reliable.

${ }^{1}$ T. Blomquist and G. Kirczenow, Nano Lett. 4, 2251 (2004).

${ }^{2}$ D. V. Melnikov and J. R. Chelikovsky, Phys. Rev. Lett. 92, 046802 (2004).

${ }^{3}$ R. A. Wolkow, Annu. Rev. Phys. Chem. 50, 413 (1999).

${ }^{4}$ R. M. Tromp, A. W. D. van der Gon, and M. C. Reuter, Phys. Rev. Lett. 68, 2313 (1992).

${ }^{5}$ L. Kipp, R. D. Bringans, D. K. Biegelsen, L. E. Swartz, and R. F. Hicks, Phys. Rev. B 50, 5448 (1994).

${ }^{6}$ Y. Wang, X. Chen, and R. J. Hamers, Phys. Rev. B 50, 4534 (1994).

${ }^{7}$ R. J. Hamers, Y. Wang, and J. Shan, Appl. Surf. Sci. 107, 25 (1996).

${ }^{8}$ N. J. Curson, S. R. Schofield, M. Y. Simmons, L. Oberbeck, J. L. O’Brien, and R. G. Clark, Phys. Rev. B 69, 195303 (2004).

${ }^{9}$ T. C. G. Reusch, N. J. Curson, S. R. Schofield, T. Hallam, and M. Y. Simmons, Surf. Sci. 600, 318 (2006).

${ }^{10}$ G. W. Brown, H. Grube, and M. E. Hawley, Phys. Rev. B 70, 121301(R) (2004).

${ }^{11}$ H. F. Wilson, O. Warschkow, N. A. Marks, S. R. Schofield, N. J. Curson, P. V. Smith, M. W. Radny, D. R. McKenzie, and M. Y. Simmons, Phys. Rev. Lett. 93, 226102 (2004).

${ }^{12}$ O. Warschkow, H. F. Wilson, N. A. Marks, S. R. Schofield, N. J. Curson, P. V. Smith, M. W. Radny, D. R. McKenzie, and M. Y. Simmons, Phys. Rev. B 72, 125328 (2005).

${ }^{13}$ M. W. Radny, et al., Phys. Rev. B 74, 113311 (2006).

${ }^{14}$ B. D. Yu and A. Oshiyama, Phys. Rev. Lett. 71, 585 (1993).

${ }^{15}$ T. L. McDonnell, N. A. Marks, O. Warschkow, H. F. Wilson, P. V. Smith, and M. W. Radny, Phys. Rev. B 72, 193307 (2005).

${ }^{16}$ T. C. G. Reusch, M. W. Radny, P. V. Smith, O. Warschkow, N. A. Marks, N. J. Curson, D. R. McKenzie, and M. Y. Simmons, J. Phys. Chem. C. 111, 6428 (2007)

${ }^{17}$ J. J. Boland, Phys. Rev. Lett. 67, 1539 (1991).

${ }^{18}$ A. Vittadini, A. Selloni, and W. Casarin, Phys. Rev. B 52, 5885 (1995).

${ }^{19}$ G. J. Xu, Surf. Sci. 577, 77 (2005).

${ }^{20}$ G. Kresse and J. Hafner, Phys. Rev. B 47, 558 (1993); 49, 14251 (1994).

${ }^{21}$ G. Kresse and J. Furthmüller, Comput. Mater. Sci. 6, 15 (1996).

${ }^{22}$ G. Kresse and J. Furthmüller, Phys. Rev. B 54, 11169 (1996).

${ }^{23}$ D. Vanderbilt, Phys. Rev. B 41, 7892 (1990).

${ }^{24}$ J. P. Perdew, J. A. Chevary, S. H. Vosko, K. A. Jackson, M. R. Pederson, D. J. Singh, and C. Fiolhais, Phys. Rev. B 46, 6671 (1992).

${ }^{25}$ J. Tersoff and D. R. Hamann, Phys. Rev. Lett. 50, 1998 (1985).

${ }^{26}$ V. G. Zavodinsky and I. A. Kuanov, Appl. Surf. Sci. 141, 193 (1999).

${ }^{27}$ D. Chen and J. J. Boland, Phys. Rev. B 65, 165336 (2002).

${ }^{28}$ V. Ramachandran and R. M. Feenstra, Phys. Rev. Lett. 82, 1000 (1999).

${ }^{29}$ M. Rolfing and J. Pollmann, Phys. Rev. Lett. 84, 125 (2000).

${ }^{30}$ M. McEllistrem, G. Haase, D. Chen, and R. J. Hamers, Phys. Rev. Lett. 70, 2471 (1993).

${ }^{31}$ R. M. Feenstra, Phys. Rev. B 50, 4561 (1994).

${ }^{32}$ G. W. Brown, H. Grube, M. Hawley, S. R. Schofield, N. J. Curson, M. Y. Simmons, and R. G. Clark, J. Appl. Phys. 92, 820 (2002). 